\title{
treasure
}

\author{
Applying modern intellectual property standards \\ to ancient medicines.
}

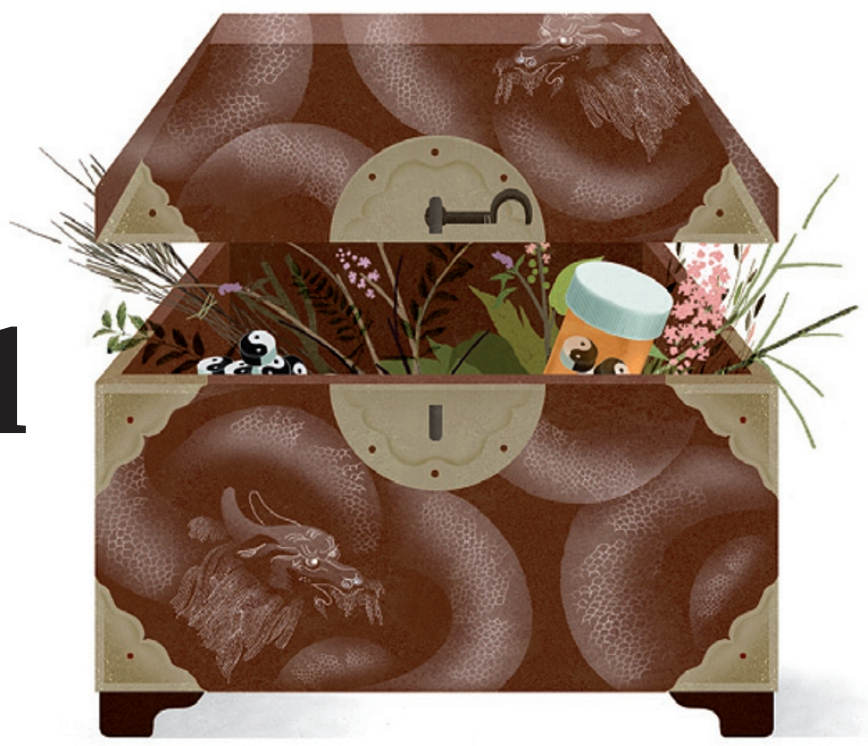

\section{BY JESSIE JIANG}

$\mathrm{T}$ he very nature of traditional Chinese medicine (TCM) poses serious challenges in patenting intellectual property. Unlike modern medicines, which are based on newly discovered, developed or isolated chemical compounds, TCM prescriptions typically consist of mixtures of well-known plant or animal extracts. Moreover, these recipes are public knowledge, having been recorded in ancient books, and are therefore ineligible for patents in areas where a compound's novelty is a prerequisite.

Even if a TCM prescription secures a patent - possibly after being safeguarded as a family secret, or as a modification to an established recipe - there are loopholes that allow the patent to be bypassed. According to TCM theories, each prescription has a fangyi, which is a description of the symptoms and an explanation of how they should be treated. A TCM prescription can maintain its fangyi even when one or several of its ingredients are replaced by similar items. For example, different species of ginseng can be used interchangeably in TCM prescriptions. This gives TCM companies leeway: under current Chinese patent law, even slight variations of a patented prescription can qualify for a new patent.

"Adding or removing an ingredient sometimes doesn't much change the efficacy of a compound TCM prescription," says Kaixian Chen, president of the Shanghai University of Traditional Chinese Medicine. This imprecision, he says, "not only leaves a lazy shortcut for patent applicants, but also undermines existing TCM patents."

Indeed, China's patent law for TCM is such that it even allows for a change in packaging design to qualify for a patent, says Xiaoting Song, a law professor at Tongji University in Shanghai who specializes in TCM intellectual property rights. "The intellectual property rights protection offered by the current patent system is weak at best," he adds.

And while TCM continues to be a source of pride for the Chinese, lax intellectual property rights create an opportunity for foreign-made medicines to enter China. According to reports

from Xinhua, China's state news agency, TCM imported from Japan, South Korea and Germany accounts for approximately $30 \%$ of the Chinese market.

China only started awarding patents for TCM products in the 1990s, and there are still no specific standards that govern whether a particular TCM formula is eligible for a patent - the same rules also govern food recipes, for example. "There is obviously an essential difference between a TCM formula and other compound products," says Song. "The concept of fangy $i$ is unique to TCM prescriptions and should be protected by the patent law." Although China's State Intellectual Property and Trademark Office is considering new standards specifically for TCM, any such policy would take years to be approved by the

"Protection offered by the current system is wealk at best." central government. Meanwhile, TCM patents for new products with little added value continue to be awarded. "My sense is that at least half of all

TCM patents in China are for packaging design rather than for the medicine itself," says Song (there are no official figures).

Despite loopholes in the patenting of TCM formulae, some global pharmaceutical companies are keen to develop new medicinal products and are expanding their R\&D efforts into the realm of traditional medicine. "Collaborations are becoming increasingly necessary when it comes to pharmaceuticals," says Song. "Even back in the 1960s, the discovery of the antimalarial artemisinin was a joint effort that involved more than 500 researchers from 60 research institutes in China."

International pharmaceutical companies submit a small fraction of TCM patent applications in China - about 3\% according to the Intellectual Property and Trademark Office. But this is set to grow. "As the pharmaceutical industry gets more competitive, TCM represents a very promising approach," says Bai Lu, vice-president of biology at the Shanghai R\&D centre of GlaxoSmithKline (GSK), the London-based global pharmaceutical company. "We are very optimistic about the TCM market." Indeed, GSK is rapidly expanding its TCM team in Shanghai, and has established collaborations with several local institutions. When it comes to new pharmaceutical agents, Lu considers the current patenting system in China to be industry friendly - companies can essentially apply for two patents for each new compound derived from a TCM formula: one for the extraction and isolation method, and a second for the end product.

Securing a patent for a new TCM product can bring great financial reward. For example, the danshen dripping pill, manufactured by Tianjin-based Tasly Pharmaceutical, is a patented cardiovascular drug that is a more concentrated and faster-releasing version of the generic danshen tablet, made from the dried root of Salvia miltiorrhiza. Since its launch in 1994, revenue from the danshen dripping pill has grown by more than $10 \%$ each year on average, reaching US $\$ 203$ million in 2010 - accounting for $28 \%$ of Tasly's total revenue.

At the same time as encouraging modernization of TCM, the Chinese government is also taking steps to preserve the traditional practice. The Chinese Ministry of Health recently approved a draft of new regulations that, if approved by the central government, will officially categorize TCM as 'traditional knowledge', a status defined by the World Intellectual Property Organization, a United Nations agency headquartered in Geneva, Switzerland. The aim of the new ministry of health regulation is to protect historic TCM prescriptions. For example, the Sijunzi decoction, a 900-year-old Chinese recipe that mixes four plants, would be recognized as traditional knowledge under the new regulation, and its derivative recipes would not be considered patentable. "TCM is our national treasure," says Song, who was a member of the drafting committee for the regulations. "It will give us the edge when competing on the world stage, and we can't afford to lose that." -

Jessie Jiang is a journalist based in Beijing. 